\title{
Ajnabiyyé bi Bourj el Barajneh ou une étrangère parmi des exilées palestiniennes
}

\author{
Roxane Caron, Ph. D.
}

Université de Montréal, Québec, Canada

Dominique Damant, Ph. D.

Université de Montréal, Québec, Canada

Catherine Flynn, Ph. D.

Université de Montréal, Québec, Canada

\begin{abstract}
Résumé
En m'appuyant sur ma démarche réflexive réalisée au cours de ma thèse portant sur l'expérience d'exil de femmes réfugiées palestiniennes au Liban, cet article propose une réflexion éthique autour de la question suivante : comment le chercheur peut-il comprendre l'Autre et porter sa voix dans le cadre d'une démarche empirique? Pour tenter de répondre, je fais un retour sur la façon dont ma position de chercheuse femme, ajnabiyyél, non musulmane, non palestinienne et engagée à "porter les voix » des femmes, s'est négociée à l'intérieur du milieu de recherche et au-delà. L'article débute par la présentation de la méthodologie de l'étude. J'y revisite ensuite les différentes problématiques qui m'ont amenée à me questionner sur ma compréhension de l'expérience des femmes palestiniennes, de ma légitimité à parler fidèlement « en leur nom » et, par conséquent, de la pertinence de mes choix méthodologiques et théoriques pour y parvenir.

Mots clés

ÉTHIQUE DE LA RECHERCHE, THÉORIES FÉMINISTES, ALTÉRITÉ, RÉFLEXIVITÉ, SUBALTERN STUDIES
\end{abstract}

«Si j'échoue dans ma tentative de transmettre leur message, je briserais la condition implicite sur laquelle ils ont offert

leurs histoires... $\rangle^{2}$ [traduction libre]

(Sayigh, 1996, p. 161).

RECHERCHES QUALITATIVES - Vol. 36(1), pp. 1-23.

VISITE DANS L'ARRIĖRE-SCÈNE DE LA RECHERCHE QUALITATIVE

ISSN 1715-8702 - http://www.recherche-qualitative.qc.ca/revue/

(C) 2017 Association pour la recherche qualitative 


\section{Introduction $^{3}$}

L'endroit grouille de monde, de bruits et d'odeurs. Les enfants courent et, curieux, me demandent en ricanant : "Chou ismik? Min wen inti? " En marchant, je croise des hommes qui sirotent le thé de même que des femmes qui me saluent : "Marhaba, ahlan wassahlan, salam alekum! $!^{5}$ » Les arômes... celui qui émane de l'échoppe à café près de chez moi... puis celui du pain de Walid, le propriétaire du forn ${ }^{6}$. Les bruits... les klaxons stridents et incessants des motocyclettes; il y a bien sûr le son maintenant familier du Allahu Akbar ${ }^{7}$. Comme je m'engouffre plus avant dans le lieu, dans ses ruelles, je trouve des commerçants prêts à me vendre fruits, légumes, tout ce qui est essentiel pour la cuisine. Impossible aussi de manquer les câbles électriques - parfois tout juste au-dessus de ma tête - qui s'entremêlent telles des toiles d'araignées. Dans ce labyrinthe où les habitations sont érigées de façon arbitraire et anarchique, chaque pan de mur est fait de graffitis, de dessins, d'affiches usées ou d'impacts de balles comme si chacun de ces symboles était le témoin d'un moment de l'histoire de cet endroit et des gens qui l'habitent...

Voilà un bref extrait de mon journal de bord qui me replonge automatiquement dans l'univers du moukhayyem Bourj el Barajneh ${ }^{8}$. Entre 2009 et 2011, j'ai fait un séjour de recherche de 18 mois au Liban, dans le camp palestinien de Bourj el Barajneh, en banlieue sud de Beyrouth. Cette année et demie a été l'une des plus difficiles de ma vie en ce qu'elle m'a fait vivre d'expériences et d'émotions. Cinq ans plus tard, il m'est toujours difficile d'ouvrir mon journal de bord ou de lire certains entretiens ... Re-vivre et re-voir Bourj el Barajneh est parfois trop pénible alors que refont surface des questionnements et des émotions reliés tantôt aux notions de privilège, de responsabilité ou de distance. Je me permets vraiment - c'est-à-dire attentivement - d'ouvrir mon journal de bord que lorsque je suis à Bourj el Barajneh, et cela, ne serait-ce que pour de courts séjours depuis $2011 \ldots$ Ce travail m'a donc conduite à m'interroger sur ma capacité comme chercheure d'écrire et de communiquer «à travers les cultures », ce qui fait maintenant l'objet des lignes qui suivent.

Si mon expérience dans le camp de réfugiés a souvent été un processus parsemé de doutes, de questionnements, de dilemmes, voire d'anxiétés propres à la recherche scientifique, interroger sa pratique de recherche et s'engager dans une réflexion critique sur les questions d'ordre méthodologique et théorique sont essentiels pour tout chercheur. De plus, comme le souligne Fainzang (2004), j'estime qu'une telle démarche n'a d'intérêt que lorsque la réflexion proposée amène une meilleure compréhension de l'Autre; compréhension qui doit systématiquement nous habiter, en tant que chercheure, afin de porter sa voix de la façon la plus fidèle qui soit. Cet article 
se veut le fruit d'un exercice réflexif articulé autour de la question suivante : comment une chercheure peut-elle comprendre l'Autre et porter sa voix dans le cadre d'une démarche empirique? Dans un premier temps, je revisiterai les différentes problématiques qui ont suscité un questionnement sur ma compréhension de l'expérience des femmes réfugiées palestiniennes de même que sur ma légitimité à parler fidèlement en leur nom. Dans un second temps, je remettrai en question la pertinence de mes choix méthodologiques et théoriques pour y parvenir.

\section{Le projet de recherche}

Cet article se base sur les résultats d'une recherche portant sur l'expérience d'exil de femmes réfugiées palestiniennes vivant dans le camp de Bourj el Barajneh au Liban (Caron, 2012). Le conflit israélo-palestinien, qui ne cesse de gagner en complexité, a largement été abordé sous l'angle géopolitique et à tel point qu'on oublie parfois qu'il $\mathrm{y}$ a des visages derrière ce à quoi l'on réfère comme «la question des réfugiés ». L'idée du projet était d'aborder cette « question des réfugiés » non pas comme un objet faisant partie d'un problème, mais plutôt de poser le regard sur les sujets et leurs expériences de l'exil et de la vie en camp de réfugiés. Cette perspective avait d'ailleurs pris racine dans mes résultats de maîtrise. À l'époque, les femmes avaient souligné leur intérêt à parler des conséquences de l'exil qui se prolongeait; un exil dans l'attente et dans l'incertitude que plusieurs femmes voyaient comme «particulier» du fait qu'il était vécu dans un camp de réfugiés, dans des conditions de vie difficiles et cela, de façon prolongée depuis plus de 65 ans... C'est donc en partant des préoccupations des femmes que mon projet de doctorat a pris forme.

\section{Mise en contexte : rencontre avec le sujet de recherche puis avec le terrain}

Mon premier contact avec la situation des réfugiées palestiniennes au Liban s'est fait au Québec, dans le couloir d'une université avec des étudiants en travail social revenant d'un séjour dans un camp de réfugiés palestiniens au Liban. Il y a de ces rencontres dans la vie qui marquent à jamais; celle-ci était de cette nature. Aux questions «pourquoi faire une recherche auprès de réfugiés palestiniens? Pourquoi ceux du Liban? » je réponds : "Parce que je cherchais à trouver un sens à quelque chose qui n'en avait pas selon moi : vivre dans un camp de réfugiés depuis plus de 50 ans à l'époque... comment est-ce possible? » Mes lectures, puis mes recherches ont donc débuté en 2005 et, encore aujourd'hui, je tente de faire sens de cette réalité... Les premières recherches documentaires sur le sujet m'ont d'ailleurs révélé une réalité complexe que je tente toujours de saisir. Et, enfin, à cette autre question souvent demandée : «Pourquoi les femmes palestiniennes?» je dirai d'emblée : «Pour leur relative absence... » Elles ne sont certes pas totalement absentes de la littérature sur les réfugiés palestiniens, mais leurs voix ne se font pas souvent entendre. J'ai donc voulu contribuer à faire affleurer leur point de vue. 
En 2009, c'est mon retour dans le camp de Bourj el Barajneh, retour pour lequel je pensais «être fin prête ». J'avais sous-estimé les défis de chaque recherche dans ce qu'elle comporte de nouvelles rencontres et de nouvelles expériences... Sans nul doute, j'avais une bonne connaissance du camp de Bourj el Barajneh, camp qui n'avait que très peu changé depuis que je l'avais quitté ${ }^{9}$. D'ailleurs, cette connaissance et cette familiarité avec le camp, ses gens, mais aussi avec le milieu et ses règles, ont grandement facilité mon retour et mon intégration. J'avais appris, lors de mon précédent séjour, qu'il était plus facile de rencontrer des participantes si je m'impliquais dans la communauté à travers notamment des activités de bénévolat, en y faisant mes emplettes journalières et en participant à des célébrations (mariages, naissances, fêtes, etc.). De plus, j'ai souvent accompagné une amie palestinienne visitant des proches ou des voisines - activité usuelle pour plusieurs femmes palestiniennes dans le camp. Je m'asseyais avec les femmes, je participais, au mieux de ma connaissance, à l'échange, et cela de plus en plus alors que ma maîtrise de la langue arabe s'améliorait au fil du temps et de mes échanges. Rapidement on me questionnait: " $\mathrm{Tu}$ es mariée? $\mathrm{Tu}$ as des enfants? Que fais-tu ici?» Puis, tranquillement, on s'intéressait à mon projet de recherche, au « récit de leur vie » que plusieurs désiraient ${ }^{10}$ me donner... Si en 2006 j'avais le sentiment de n'être, du moins pour certaines, qu'une autre occidentale venant observer leur vie, leur poser des questions pour ensuite quitter, ce sentiment n'était plus aussi dominant en 2009... mon statut avait changé parce que j'étais revenue. Ce «retour», auquel s'ajoutait le fait d'habiter le camp, semblait important pour plusieurs femmes.

\section{Approche méthodologique}

Pour cette étude, j'ai privilégié la recherche qualitative puisqu'elle permet de se concentrer sur l'expérience subjective des femmes et sur les significations qu'elles attribuent à celle-ci (Maynard, 1994). Le leitmotiv du projet étant l'intérêt pour l'expérience d'exil de ces femmes, il m'importait de saisir le sens qu'elles leur donnaient. Aussi, en témoignant, les femmes feraient ainsi «acte d'auteur», pour paraphraser Giorgio Agamben (1999, p. 176). Cette étude s'inscrit également dans une perspective ethnographique par sa volonté de développer une connaissance " intime» du milieu de la recherche. Enfin, le cadre d'analyse, le féminisme postcolonial, exige de la chercheure une réflexion constante sur elle-même en ce qu'elle représente pour l'Autre et en tenant compte des rapports de pouvoir en présence dans la relation. Conséquemment, trois méthodes ont été conjuguées: l'observation participante, l'analyse documentaire et l'entretien semi-structuré de type récit de vie.

L'observation participante ${ }^{11}$ a notamment contribué à la description de l'environnement qu'est le camp de Bourj el Barajneh de même que des événements qui s'y déroulent. Elle m'a permis de développer une connaissance «intime» du milieu. Un des moyens de partager « intimement » les expériences des femmes est de créer des 
liens avec des femmes, des hommes et des enfants du camp de Bourj el Barajneh. En dehors de mes entretiens officiels, j'ai donc passé de nombreuses heures en compagnie de voisins, de familles, de certaines femmes du camp avec qui j'ai développé des amitiés. Cette proximité m'a donné accès à leurs réalités, leurs difficultés, leurs espoirs, leurs moments de doute, mais aussi leurs moments heureux (naissance, fiançailles, etc.). Vivre dans le camp m'a aussi permis de multiplier les occasions de rencontres informelles où j'ai discuté, d'une façon ou d'une autre, de mon sujet d'étude tantôt lors d'un repas, d'une visite pour le thé ou lors d'une conférence. Mon journal de bord m'a permis de consigner mes expériences, mes activités quotidiennes et mes observations. Le chercheur n'étant pas un observateur scientifique neutre et rationnel, mon journal est devenu un espace pour noter mon sentiment d'impasse par rapport à ma propre personne, à mon statut, à ma compétence comme chercheur, à ma responsabilité morale liée à la relation que j'entretenais avec celles que je tentais « d'étudier »; les conséquences aussi de mon travail, ses suites... Une bonne partie de ces « efforts ethnographiques » se retrouvent dans les lignes de cet article.

L'analyse documentaire des textes du droit international et national libanais à l'égard des réfugiés palestiniens a quant à elle enrichi ma compréhension de l'expérience d'exil des femmes, grâce notamment à une connaissance en profondeur des législations et droits relatifs à leur statut de réfugiées et d'apatrides.

Enfin, l'entretien de type récit de vie a permis la mise en forme de l'expérience d'exil des femmes : parcours, expériences, difficultés, stratégies, priorités, etc. Au total, 42 Palestiniennes âgées de 21 à 82 ans ont participé à la recherche. Ces femmes ont été recrutées avec l'aide d'organisations non gouvernementales (ONG), de personnes-ressources dans le camp de Bourj el Barajneh, de même que par des participantes ou des femmes qui me demandaient si elles pouvaient participer à la recherche. Pour refléter une diversité d'expérience, j'ai rencontré des femmes différentes en ce qui a trait, notamment, à leur vécu en Palestine, à leur âge, au nombre d'années de vie dans le camp de Bourj el Barajneh, au statut social (mariée ou non, séparée, divorcée, veuve), à la situation familiale (avec ou sans enfants) et au milieu socioéconomique. Alors que quelques études (Holt, 2011; Latif, 2008) ont sollicité la parole des femmes du camp de Bourj el Barajneh, je me suis assurée de recruter des femmes qui n'avaient pas encore eu l'occasion d'être entendues. Les entretiens ont été menés en arabe et la contribution d'une interprète a été nécessaire pour la traduction de l'arabe à l'anglais. Mes connaissances de l'arabe dialectal (intermédiaire) ne m'ont pas permis de conduire les entretiens seule, bien que j'arrivais à saisir des éléments du discours dans la langue originale. Au fil de mon séjour, je saisissais de plus en plus le contenu des récits et donc, ma compréhension de l'entretien s'est améliorée. Des entretiens avec d'autres acteurs de la communauté ont aussi permis le croisement des informations et des points de vue: rencontres avec des groupes politiques, des représentants de l'UNRWA ${ }^{12}$, quatre organisations locales offrant des services aux 
femmes de la communauté, un médecin, une travailleuse sociale et d'autres personnesressources, tel un homme souvent interpellé lors de conflits qu'on m'a présenté comme le sage de la communauté. L'idée de faire un groupe de discussion avait été envisagée puis mise de côté. Craignant - selon mes expériences précédentes dans le camp lors de mon premier séjour - que participer à la recherche soit une affaire du domaine du privé, j'ai privilégié l'entretien individuel. Étant " étrangère » dans le camp, est-ce qu'on allait participer à une rencontre de groupe pour échanger sur les préoccupations, besoins, etc., des femmes? J'ai ainsi opté pour une autre approche. Accompagnée de mon interprète, j'ai rencontré des groupes de femmes fréquentant des organisations du camp afin de présenter mon projet de recherche (but, objectifs, méthode par récit de vie). Après la rencontre, j'avais l'habitude de rester et de participer aux activités de l'organisme (préparation de repas, groupes de discussion, artisanat, etc.); une telle approche permettait aux femmes présentes de poser des questions sur le projet, mais aussi sur les raisons de ma présence dans le camp, et contribuait ainsi à établir un lien de confiance.

\section{Cadre d'analyse}

La mémoire palestinienne, dont le vécu pré-1948 et la nakba ${ }^{13}$, a longtemps été, dans son ensemble, occultée par un récit sioniste dominant. Si des historiens ont grandement contribué à reconstituer la mémoire collective palestinienne, la spécificité de l'expérience des femmes et la façon dont leurs récits sont construits par le genre ont peu été étudiées ${ }^{14}$. Certains auteurs (Hasso, 2000; Sayigh, 2007a) expliquent cet état de fait par l'idée qu'on croit tantôt que les femmes palestiniennes ne sont pas des sources fiables pour raconter l'histoire et la dépossession, ou encore que leurs récits déstabilisent et compliquent les récits politiques déjà établis. Considérant que cette étude s'inscrit dans une lignée de travaux qui rendent leur juste place aux «voix silencieuses » que sont souvent celles des femmes réfugiées palestiniennes des camps, je me suis inspirée des travaux des féministes postcoloniales (Kandiyoti, 1996; Mohanty, 1988; Spivak, 1988) et des féministes noires américaines (Collins, 2000; Crenshaw, 1991).

\section{Comment la chercheure peut-elle comprendre l'Autre et porter sa voix dans le cadre d'une démarche empirique?}

La question de Spivak (1988) «Est-ce que les subalternes peuvent parler? $»^{15}$ [traduction libre] a accompagné mes réflexions. Appliquée à mes recherches, cette question s'est articulée autour de différentes interrogations : est-ce que je saisis bien les réalités des femmes? Est-ce que ce que j'écris témoigne de leurs réalités? Puis-je « vraiment» parler en leur nom? Le cas échéant, à quelles conditions dois-je le faire? Ces questionnements ont été porteurs de défis pour la réalisation même de cette étude dans la mesure où la linéarité du processus de la recherche a été remise en question au profit d'une démarche engagée et dialogique. Les allers-retours entre les analyses et les 
échanges avec les participantes se sont articulés dans un souci d'élargir le processus de restitution des données au-delà de la finalité de la recherche et ont contribué à construire un sentiment de proximité et d'intimité avec les participantes. Pour tenter de répondre aux questions qui me taraudaient lors de l'écriture et relever les différents défis qui y sont associés, j'amène ici le lecteur à revivre le processus réflexif parcouru.

\section{Des questionnements dans le milieu de la recherche}

Je suis entrée dans le milieu de recherche - le camp de Bourj El Barajneh - avec le fervent désir de comprendre la réalité des femmes qui y vivaient. J'étais consciente et convaincue que la recherche devait correspondre à leur définition de la réalité. Ainsi, ma décision d'utiliser une approche féministe entraînait un certain nombre d'obligations et de défis, d'abord envers les femmes rencontrées, mais aussi envers la communauté académique à laquelle j'appartiens. En effet, outre la situation des femmes, la recherche féministe s'intéresse, comme le précisent Holland et Ramazanoglu (2002), à la façon dont les connaissances sont produites : les féministes se sentent une "responsabilité morale» liée aux connaissances que leurs travaux permettent de dégager et cela demande l'application d'une éthique stricte envers les communautés de femmes auprès desquelles elles interviennent. Cette responsabilité envers les femmes qui ont participé à ma recherche, je l'ai bel et bien ressentie. Pour «porter des voix », je dois les saisir, les comprendre. Or, si ma position de chercheure féministe valorise le point de vue des femmes, comment m'assurer que ce dernier est respecté? Comment puis-je contribuer à présenter l'expérience des femmes réfugiées palestiniennes sans «distorsionner» leur voix? Ces interrogations rejoignent en tout point les préoccupations de Lorraine Code voulant que « l'aspect politique de la prise de parole pour d'autres femmes, à leur propos et en leur nom, est l'une des zones les plus contestées de l'activisme et de la recherche féministes d'aujourd'hui » (citée dans Rose, 2002, p. 29). Ces considérations m'étaient importantes d'autant plus que nombre de participantes semblaient parler «au monde» à travers moi. En effet, plusieurs d'entre elles m'ont confié qu'elles espéraient, par leur témoignage, que soient entendues leurs réalités de femmes palestiniennes, leurs expériences de femmes réfugiées vivant dans un camp depuis des générations et leurs identités de femmes musulmanes. Donc, elles acceptaient de me parler avec ce désir. En quelque sorte, cela ne renforce-t-il pas ma responsabilité de chercheure?

Cette question soulève inévitablement des doutes quant à ma légitimité en tant que chercheure outsider ${ }^{16}$. Mais l'opposition chercheur outsider et chercheur insider est-elle un faux débat? Dans un contexte où les femmes m'ont explicitement demandé de «porter leur voix», la responsabilité de bien les comprendre m'est apparue particulièrement importante. Or à quoi sert de porter leur voix si je déforme leurs propos? Parler de l'Autre, témoigner «au nom de l'Autre», implique de préciser « d'où on parle ». Il s'agit de réfléchir à la façon dont ma propre expérience est 
susceptible d'influencer ma compréhension du vécu de ces femmes. Ainsi, cela implique de définir mon positionnement social. Mais comment me positionner? Par rapport à qui? À quoi? Mon expérience de recherche m'a révélé le caractère plutôt fluide et changeant de mes multiples identités.

Basée sur cette fluidité, Anthias (2005) avance que le positionnement social réfère aux processus par lesquels les individus participent à la construction de leur identité. À l'aide d'un exemple tiré de l'expérience de femmes immigrantes, Harper (2012) note différentes actions par lesquelles le positionnement social s'articule. Elle propose, entre autres, que celui-ci se construit à travers la façon dont les femmes se racontent et négocient leur identité dans leurs interactions avec les différents acteurs qu'elles côtoient, dans leur engagement au sein de la communauté à laquelle elles s'affilient, de même que dans différents projets politiques. En transposant cet exemple à ma situation, mon premier réflexe a été de définir mon positionnement social par rapport au milieu de recherche dans lequel je me suis engagée. J'ai donc construit mon identité comme étrangère, non palestinienne et non musulmane. Or cette «posture de négation » émerge de ma construction identitaire par rapport à la façon dont les femmes rencontrées se sont positionnées dans leur récit. Ainsi, je me suis positionnée d'emblée comme n'étant pas l'une d'elles, position manifestée par le qualificatif de ajnabiyyé souvent murmuré sur mon passage ou par certaines participantes qui me disaient avoir entendu parler de la présence de cette ajnabiyyé dans le camp. En fait, je m'identifiais comme tout ce que je n'étais pas, soit Palestinienne, musulmane et réfugiée. Or est-ce que je pouvais «bien» les comprendre n'étant pas comme elles à plusieurs égards? Considérant qu'on ne peut jamais totalement saisir comment notre présence est perçue par la population du camp, je suis retournée rencontrer certaines femmes et leur ai notamment posé la question suivante : «Pourquoi m'avez-vous parlé alors que je suis une étrangère, non palestinienne, non musulmane, non réfugiée? Voici les réflexions de quelques-unes d'entre elles ${ }^{17}$ :

Premièrement, parce que nous sommes tous des êtres humains; nous avons tous été créés par Dieu. Deuxièmement, tu es une personne très respectable. Quand tu es venue me voir, tu m'as posé des questions de façon aimable donc j'ai répondu à tes questions. Encore une fois, je ne me soucie pas de ta religion ou de ton histoire, la façon dont tu as agi était plus importante pour moi... et je suis certaine que tu diras ce que tu as vu et vécu ici dans le camp [...]. Je suis sûre que, maintenant que tu retournes dans ton pays, que tu ne vas parler en mal de moi parce qu'il y avait du respect entre toi et moi... Je suis certaine que tu vas parler de «notre cause », de notre peuple, de notre vie ici, le camp, et je suis sûre que tu diras toutes ces choses à ton monde, à ton peuple (Hayat, 62 ans). 
Tout d'abord, parce que t'es un être humain comme moi, que t'es pas différente; t'es pas une « chose » ou un mur! [...] Peut-être que les gens ne liront pas ta thèse en ce moment, mais plus tard dans la vie, une génération, une génération qui ne sait pas à propos de nous, et cette personne va lire ce que t'as vécu ici, ce que tu as écrit... donc j'apprécie la mission que tu t'es donnée, tes manières, et je t'apprécie comme être humain (Sou'ad, 72 ans).

J'ai accepté de te parler parce que, peut-être, que tu ne peux pas m'aider personnellement, mais tu peux parler à d'autres personnes au sujet de ma situation difficile ici [...] Je veux que les gens sachent que nous existons, qu'ils sachent notre situation... et je crois que tu es une personne très honnête et que tu n'es pas venue ici pour nous tricher ou pour profiter de nous... Je veux dire, «nous utiliser »... et de toute façon, si tu étais une tricheuse, je ne pense pas que tu aurais pu rester avec nous aussi longtemps. [...] Je sais que tu as quitté ton pays, tes coutumes, tes traditions pour venir ici. Tu t'es adaptée à nos conditions et modes de vie [...]. Donc, tout ce que nous vivons ici, tu le vis aussi! Tu as aussi intégré ces choses à ta vie, je veux dire nos coutumes... c'était une bonne chose à faire pour nous (Basma, 41 ans).

Les réponses de ces femmes reflètent le lien qui s'est créé entre elles et moi, à travers ma présence dans le camp, à travers leur quotidien. C'est toutefois plus que ma présence, mais le fait de partager leur réalité en vivant dans le camp avec elles : vivre dans un environnement insalubre avec les interruptions d'électricité fréquentes, avec l'eau saumâtre impropre à la consommation dans les habitations. Vivre et partager le quotidien des femmes, mais aussi, comme plusieurs me le disent lors des entretiens, respecter et participer à plusieurs moments de leur vie. Donc, non seulement vivre et reproduire ces gestes du quotidien, mais m'engager à tenter de comprendre leur signification et leur importance. C'est tout cela qui m'a fait passer d'une position de négation externe à une autre position, soit celle d'être considérée comme l'une des leurs : de outsider je suis passée à insider. Ceci dit, cette position ne doit pas être tenue pour acquise, elle doit sans cesse être renégociée auprès de nouvelles femmes que je rencontre, au fil des réalités dans le camp qui changent, etc. Comme le précise Sayigh (1996), si on est en quelque sorte « adopté » par le milieu, par une famille du camp par exemple, cela nous transforme : de visiteur, on devient protégé à quasi-membre. Si j'ai effectivement vécu cette situation avec mes voisins proches, je pensais tout de même rester, pour nombre de participantes, une outsider, une ajnabiyyé. En fait, je me suis longtemps perçue essentiellement comme outsider alors que plusieurs de leurs témoignages ont plutôt montré que j'étais maintenant insider. Selon Harper (2012), c'est autour d'éléments tels que l'engagement et l'attachement que le positionnement social peut s'articuler. 
Lors des entretiens rétroactifs, les femmes ne soulignent pas tant nos différences que ce qui nous lie, le lien qui s'est créé; le respect et la confiance qui se sont établis. Comme le souligne Guillaumin, penser en termes de "différences », soit en ce qui nous différencie, nous place inévitablement « dans un rapport d'un type particulier où il y a un point fixe, un centre qui ordonne autour de lui et auquel les choses se mesurent, en un mot, un référent » $(1992$, p. 97). Ainsi, maintenir ma dynamique avec les femmes sur la base des différences peut renforcer un sentiment d'extranéité et une telle attitude peut maintenir des rapports dominants-dominés, «ce qui nous met en mauvaise posture pour les combattre et les détruire » (Guillaumin, 1992, p. 99). Je ne crois pas que l'on doive écarter complètement le concept de différence, mais du moins rester attentif à ses limites... Plusieurs femmes réfugiées palestiniennes me les ont d'ailleurs rappelées.

Lors de mes entretiens rétroactifs, j'ai échangé avec des participantes afin de leur faire part de mes analyses préliminaires ${ }^{18}$, l'idée étant de partager le fruit de ces analyses et d'engager un dialogue avec elles. Il est important ici de mettre l'accent sur le terme dialogue dans la mesure où ma volonté était de créer un espace interactif: j'étais déterminée à éviter un échange d'informations factuelles, deux monologues où chaque personne expose sa pensée sans tenir compte des remarques, des critiques, des réserves, etc., de l'autre. Certes, le risque d'un tel exercice réside dans le fait que certaines femmes peuvent se sentir intimidées ou inconfortables à émettre des commentaires, à apporter des critiques. Mais celles-ci n'ont pas été silencieuses! Conséquence de cette étape : les dialogues ont permis de valider certaines de mes analyses, ont apporté davantage de précisions et ont levé des ambigüités. En définitive, ils ont permis de mieux saisir les réalités des femmes. À titre d'exemple, ces entretiens ont permis de clarifier le rapport qu'entretiennent certaines femmes à l'islam. D'emblée, elles sont plusieurs à lier leur besoin d'affirmation religieuse aux souffrances engendrées par les quinze années de guerre au Liban, mais aussi aux souffrances de l'exil et de la vie en camp de réfugiés qui durent, pour nombre d'entre elles, depuis plus de 65 ans. À la lumière de ces échanges, j'ai pu comprendre l'importance de la religion pour plusieurs femmes : elle donne un sens à leur vie. De façon réciproque, ces mêmes entretiens ont eu un effet semblable chez les participantes puisque, de leurs propres témoignages, elles m'ont dit porter un regard différent sur leur réalité. En effet, nos entretiens ont permis à plusieurs participantes de réfléchir à l'ambivalence qui caractérise leur rapport au camp : on « déteste » ou on « rejette » le camp pour ces conditions de vie intenables, mais on y est « attaché » pour tout ce qu'il représente comme liens, souvenirs, histoires, etc.

Je crois que ces entretiens rétroactifs ont été à ce point révélateurs - percutants, parfois - que je ne referais plus de recherche autrement. Si ces rencontres m'ont permis de valider ou nuancer certaines analyses, elles ont surtout modifié la dynamique participante-chercheure. Nous n'étions plus dans une relation de donneur-récepteur, 
mais plutôt dans un rapport de coconstruction où il $\mathrm{y}$ avait un va-et-vient d'informations, d'analyses et de questions entre les deux parties. Plusieurs m'ont confié avoir déjà participé à de nombreuses recherches, mais sans jamais avoir contribué à l'étape de l'analyse des résultats. La création de cet espace de discussion a permis aux femmes de participer à la définition de leur réalité. Comme le précise Collins (2000) : «Se définir est la première étape vers l'autonomisation : si un groupe ne se définit pas lui-même, il se voit définit par l'Autre et pour les besoins de l'Autre ${ }^{19}$ [traduction libre] (p. 101).

J'avais la conviction que les questionnements soulevés au milieu de ma recherche étaient résolus, mais voilà que, de retour au Québec, ils surgissaient à nouveau. Ce sentiment de doute par rapport à mes analyses m'a rappelé la métaphore de Siran (1994) : « je croyais avoir en main un galet alors que la réalité me filait entre les doigts... » (p. 113).

\section{Des questionnements à l'extérieur du milieu de recherche}

Une intimité se crée à vivre au quotidien pendant un an et demi dans un milieu, une intimité avec ses lieux, ses odeurs, ses activités. Initialement, je devais compléter le processus d'écriture et d'analyse au Liban. Je prévoyais quitter le camp pour habiter plus près du centre de la capitale libanaise, l'idée étant de prendre du recul par rapport à mon milieu de recherche. Toutefois, j'avais négligé la difficulté qu'une telle rupture impliquerait : quitter le camp pour habiter "à l'extérieur», un extérieur lourd de significations. Cela signifiait «abandonner » les Palestiniennes et me mettre dans une position privilégiée; une position que je me serais accordée en me prévalant d'un droit qui leur est refusé : celui de choisir sa situation. J'ai donc décidé de revenir au pays, d'être chez moi et non pas «à l'extérieur du camp ». Si une telle décision me semblait justifiée, je n'avais pas prévu ses conséquences. En fait, j'avais envisagé la rupture émotive avec le milieu, ses gens, mes amitiés, etc., mais l'inattendu s'est présenté sous la forme d'une autre rupture, celle-là avec les participantes.

Une fois le lien coupé avec le camp de Bourj el Barajneh et le Liban, la peur de trahir la voix des femmes s'est intensifiée de nouveau, et cela, au point de ne plus me permettre de prendre position sur les différents rapports de pouvoir vécus par ces femmes, principalement sur les rapports de genre. Je me suis muselée pendant une longue période. En effet, devant l'absence des femmes pour réagir à mes analyses, j'avais l'impression d'avoir une position hégémonique à leur égard. Ainsi, je me retrouvais au Québec, dans la même situation que celle vécue vers le milieu de ma recherche, tourmentée par des questionnements qui surgissaient de nouveau alors que je les croyais déjà résolus : «À quoi bon dire ce que je vois, ce que je comprends, etc., alors que j'essaie de faire émerger comment elles voient, comment elles perçoivent leur situation?»J'ai donc (re)plongé dans une réflexion sur mon rôle comme chercheure: dois-je n'être qu'une «porteuse de messages » et me museler pour ne 
laisser paraître que les propos des femmes? Les entretiens rétroactifs s'étaient déroulés de telle sorte qu'un espace de discussion s'était ouvert où chacune d'entre nous exposait et discutait de ses idées. Je m'étais d'ailleurs permis d'être directe en abordant des sujets délicats qui mettent en évidence la différence des perceptions (par exemple les formes de violence). Mon retour au Québec m'a toutefois coupée de la dynamique dialogique récemment créée avec les femmes et je me suis en quelque sorte repliée sur moi-même.

Toutes ces questions qui m'ont accompagnée, voire traquée au cours de ce processus n'ont pas été veines. La façon dont on présente les choses est capitale : il importe de s'interroger sur notre façon de rendre compte des expériences de ceux et celles avec qui nous avons vécu. L'étape de rédaction n'est jamais « un simple exercice de transcription ou de décodage, mais un exercice de construction et de traduction au cours duquel le chercheur produit plus qu'il ne reproduit » (Ghasarian, 2004, p. 18). De plus, nos écrits s'enracinent dans des relations de pouvoir (Ghasarian, 2004). Je n'ai donc jamais oublié que comme "chercheure détentrice de la plume et du papier », je tiens une position d'autorité incontestée (Ghasarian, 2004). Il ne tient qu'à la chercheure-écrivaine de «traquer l'ethnocentrisme occidental, [de] démasquer les jugements subjectifs, [de] faire apparaître les conséquences des préjugés, etc.» (Godelier, 2004, p. 194).

Désormais, à travers mon travail d'écriture, je cherche à créer, à l'instar de Rabinow, « un produit hybride et transculturel» (1988, p. 137-138). Pour ce faire, j'estime que le travail dans le milieu de recherche doit servir à « élaborer un système de symboles partagés si on veut mener à terme le processus de constitution d'un objet par la réflexion, l'objectivation, la représentation et l'explication » (Rabinow, 1988, p. 137-138). Un chercheur doit constamment faire une réflexion sur lui-même, en particulier lorsque ses travaux s'inscrivent dans un milieu culturel différent; il doit

s'observer de l'extérieur pour comprendre comment [il] peut apparaître à des personnes issues de milieu[x] radicalement différent[s] et pour pouvoir examiner ses propres préjugés culturels et historiques [...] Le véritable défi à relever est de parvenir à faire œuvre de [traducteur, de facilitateur ou d'intermédiaire] (Rose, 2002, p. 38).

Si je suis d'accord avec cette position, ma recherche n'est pas participative, au sens strict du terme, puisque les femmes n'ont pas entièrement le contrôle du projet. Ceci dit, je continue de penser que « l'espace de discussion » est intéressant à explorer en ce qu'il laisse un espace aux deux voix. Le défi ici est donc de leur accorder une place égale.

Mon processus réflexif ne s'est pas arrêté ici. Forte de mon expérience de recherche - de ses surprises, de ses doutes, de ses découvertes, de ses pistes -, je suis allée au-delà de ma pratique d'enquête et d'écriture pour réexaminer mes choix... 


\section{Des questionnements qui se poursuivent}

Alors que des perspectives féministes comme le féminisme postcolonial ont dénoncé l'anhistoricité et l'universalité du discours de sororité des femmes, discours dominant d'un certain féminisme, l'analyse des récits de vie des participantes a tout de même dégagé une nette propension de la part de plusieurs participantes à utiliser le pronom «nous" pour livrer leur témoignage. En effet, certaines mettaient l'accent sur l'expérience collective des Palestiniens des camps et n'utilisaient le « je » que pour appuyer d'un exemple le drame commun des réfugiés palestiniens des camps au Liban. Mais si ce «nous» n'était pas le «nous, les femmes" du féminisme occidental dominant, il était assez important dans mon corpus de récits pour que je réfléchisse au sens à donner à la prédominance du «nous » sur le « je» chez ces femmes. Aussitôt cette réflexion a-t-elle émergé que je me suis de nouveau engagée dans un va-et-vient, examinant les récits des femmes tout en me questionnant sur mes propres motivations pour une telle question. Au terme de ma réflexion, j'en suis arrivée aux interrogations suivantes : qu'est-ce que la mise en mots des récits des femmes - tantôt au «nous » tantôt au «je»- révèle des identités qui construisent leur vie? Qu'est-ce que cette question - soit cet intérêt pour le « je »-dit des identités que moi-même je priorise? Tenter de trouver des réponses à ces deux interrogations m'a amenée à explorer deux pistes : la méthodologie utilisée et le cadre théorique retenu.

\section{Une méthodologie appropriée?}

D'abord, je me suis (re)posée la question de savoir ce qu'est le récit de vie. Selon Orofiamma (2008), cette méthode a pour visée de rendre compte de l'expérience d'un sujet et du contexte (au moment où le récit est fait); elle a ceci d'intéressant que, à travers l'exercice, chaque personne tend à construire les identités par lesquelles elle souhaite se faire reconnaître.

Donc le «nous », c'est ce qui les rattache aux autres - aux membres de la communauté, à la famille -, c'est la femme dans son histoire collective. Or les femmes réfugiées palestiniennes ne parlent pas d'un «nous» qui réfèrerait à un «nous, la communauté de femmes », tel que vu par un certain féminisme libéral dominant, mais bien à un «nous » qui a longtemps été effacé ou non reconnu, soit «nous, le peuple palestinien », une communauté dont on ne reconnaît que trop peu les droits, voire l'existence. Malgré tout, comme Sayigh (1996), je craignais que « la crise nationale et le discours nationaliste dominent le récit des femmes; à un tel point que leur expérience de genre soit éliminée de leur discours $»^{20}$ [traduction libre] (p. 159). Si ce « nous » structure la grande majorité des récits - à travers notamment la lutte nationale palestinienne -, le «je» n'est pas absent des récits, bien au contraire, alors que plusieurs femmes témoignent de leur parcours personnel lié tantôt à leur vie amoureuse, à leur scolarité ou encore à leurs croyances religieuses. 
Mais cette recherche du «je » dans les récits n'est-elle pas révélatrice de ma propre identité comme chercheure? Mes questionnements et mes analyses reflètent la complexe «nature » de nos identités - la mienne et celles des participantes -, identités qui se composent d'éléments divers selon notre contexte social, culturel, historique et politique. Le fait de «chercher le je » révèle, notamment, ma propension à mettre l'individu au cœur de mon récit de vie alors que plusieurs des réfugiées palestiniennes mettent plutôt la collectivité au centre du leur.

Par ailleurs, il importe de souligner que le pronom «nous », si présent dans les récits, apparaît comme révélateur des luttes tant politiques qu'idéologiques des femmes. À cet effet, j'ai constaté que les femmes étaient actrices de la transmission de la mémoire, de l'éducation et de l'identité religieuse. Or ces luttes se jouent à la fois sur le plan collectif et sur le plan personnel. Prenons l'éducation pour exemple : les femmes en parlent au «nous» comme d'une lutte à mener pour la communauté palestinienne autant que pour les femmes en tant que groupe social. Mais certaines en parlent aussi comme d'une lutte à poursuivre au plan personnel, et ce, bien que leurs objectifs de scolarisation aillent à l'encontre des normes traditionnelles de genre. Enfin, cet usage du « nous » et du « je » n'est-il pas révélateur d'un désir d'affirmation personnelle des femmes, c'est-à-dire d'un désir de passer, comme le formule De Gaulejac (1987), de "produit » de l'histoire à « sujet» de l'histoire? Les femmes reconnaissent les liens d'appartenance à la communauté, certes, mais elles affichent aussi des choix personnels qui tissent en quelque sorte la trame singulière de leur existence.

Enfin, plutôt que de chercher à circonscrire le « je», il importe de prendre le récit pour ce qu'il est. Je pense avoir réussi à dégager, à travers ces récits à la fois au « nous » et au «je », ce qu'ils avaient à révéler sur les femmes, sur leur vision du monde, leurs priorités et leurs luttes. Abu-Lughod (1993), dans son étude auprès de femmes bédouines en Égypte, apporte toutefois des réserves, voire des objections, à utiliser le récit de vie comme outil méthodologique avec certaines communautés puisque «le récit de vie peut créer le sentiment que la personne apparaît comme "le" centre du récit ou encore, comme un individu isolé ${ }^{21}$ [traduction libre] (p. 31); or, selon elle, les femmes bédouines ne se perçoivent pas comme telles. De la même façon, plusieurs Palestiniennes ne se perçoivent pas comme des individus isolés.

Le récit de vie comme choix méthodologique amène-t-il des contraintes aux sujets, imposant une façon particulière de se raconter? Une des participantes de mon étude, Rana, que j'ai surnommé « la chanteuse », a livré son récit en l'entrecoupant de chansons qui illustraient son parcours. Bien que cette forme m'ait déroutée et que Rana ait été l'unique participante à l'adopter, je l'ai laissée se raconter et « se chanter » en m'efforçant de m'adapter à ce type de récit. J'en retiens qu'il y a une réflexion à mener sur le caractère hégémonique du récit de vie essentiellement chronologique ${ }^{22} \ldots$ Cette 
propension que j'avais à chercher le « je » dans les récits des femmes n'est-elle pas liée au discours émancipatoire individuel des femmes comme «première » lutte des groupes de femmes? S'agit-il de la valorisation chez moi d'une forme de récit plutôt qu'une autre? En fait, est-ce moi qui impose une forme de récit alors que les femmes ont une autre façon de se raconter et de mettre en mots leur expérience? Ces questionnements en progression m'ont conduite à poursuivre ma réflexion, cette fois sur mes ancrages théoriques...

Une grille de lecture théorique appropriée?

La théorie féministe postcoloniale s'avérait un choix approprié afin de comprendre les réalités des femmes réfugiées palestiniennes. Selon Humm (1995), cette analyse est pertinente lorsqu'on s'intéresse à la vie de femmes provenant de certains groupes minoritaires. L'histoire sociale des réfugiés palestiniens étant largement restée dans l'ombre - en particulier celle des femmes des camps (Latte Abdallah, 2004) -, placer l'expérience des femmes palestiniennes au centre de l'analyse, c'était faire une place à leurs voix, à leurs définitions du «monde». De plus, une grille de lecture féministe postcoloniale pose un regard critique sur les schémas occidentaux de libération individuelle des femmes comme lutte première de «toutes» les femmes. Par conséquent, son articulation dans le cadre de ma recherche permettait l'analyse de l'intersection des luttes liées au genre et celles liées à l'histoire coloniale, à la pauvreté, à la lutte nationale ainsi qu'à d'autres luttes émergeant du discours des Palestiniennes. De ce fait, cette approche théorique me donnait l'avantage de consigner les réalités des femmes réfugiées dans un prisme élargi, inscrivant les luttes de genre au croisement et non au centre - d'autres luttes privilégiées par les Palestiniennes. Or l'application du féminisme postcolonial m'a conduite vers une impasse...

Un des éléments qui a alimenté le développement du féminisme postcolonial a été la dénonciation de l'hégémonie d'un certain discours féministe occidental, plus précisément de schémas « universalisant », et de l'image de victimes qu'il donne des « autres » femmes. En réponse à cette image, la théorie féministe postcoloniale prône "l'hétérogénéité » des expériences et des façons de se définir des femmes. Ainsi, j'avais choisi ce cadre théorique notamment parce qu'il reconnaît aux femmes la possibilité d'une pluralité de façons de se définir. Or, par sa dénonciation trop vive de l'image de victime véhiculée par un certain féminisme occidental, l'application d'un féminisme postcolonial semble favoriser l'émergence d'une figure tout aussi dominante, soit celle d'une femme résistante ou combattante. Cela rejoint les critiques de Bilge (2010), qui note que si l'analyse féministe libérale/universaliste crée une image de «soumission», à l'opposé, c'est une image de «résistance» que l'analyse féministe postcoloniale crée. Or l'inconvénient de schémas dichotomiques (femmevictime - femme-combattante) est d'omettre toutes les autres réalités de femmes qui se situent entre ces deux pôles. J'admets toutefois qu'il est facile de tomber dans ce piège 
qui se manifeste souvent par un effet de pendule : si on me parle des femmes arabomusulmanes comme des femmes victimes de l'oppression masculine, par exemple, j'ai le réflexe de présenter l'image contraire, soit celle d'une combattante, d'une résistante du quotidien. Ce va-et-vient entre des positions d'attaque et de défense ne permet pas, selon moi, de saisir les réalités nuancées des femmes. Celles des Palestiniennes sont en effet plus complexes que cette représentation binaire le laisse croire. Toutefois, le féminisme postcolonial ne devrait pas, théoriquement, présenter uniquement cette image de la femme résistante. En effet, il est question, dans les écrits fondateurs de l'approche, de l'hétérogénéité des réalités; serait-ce donc dans l'application de cette théorie que l'hétérogénéité se perd? Je me demande si la réponse ne se trouve pas dans cette question de Gayatri Spivak, auteure phare du féminisme postcolonial : «les subalternes peuvent-elles parler? $\gg^{23}$ [traduction libre] Cette question m'a taraudée lors de l'analyse de mes résultats alors que je cherchais à ce que toutes les visions des femmes soient présentées, tous les discours entendus. Mais en me questionnant de la sorte, je cherchais constamment des récits de femmes "qu'on entendait peu», je cherchais des discours minoritaires. Un tel exercice a eu pour effet de reconduire les pôles victime-résistante. Cet effet pendulaire était tel que si je voyais une femme dans une position de victime, je cherchais à voir si elle n'était pas actrice dans une autre dimension de sa vie. Ce qui rejoint à nouveau Bilge :

Avec leur empressement à « donner une voix » et à inspirer l'activisme en trouvant ou réinventant les traditions de résistance, les approches orientées vers la résistance, biaisées par un certain romantisme, appauvrissent considérablement l'analyse du pouvoir - les chercheurs étant trop préoccupés à trouver «des résistantes » et pas assez attentifs à expliquer les rouages du pouvoir. Il est donc nécessaire de maintenir l'analyse intersectionnelle intégrée aux analyses postcoloniales, mais tout en dissociant l'interprétation de l'agentivité des femmes voilées de la doxa de la résistance ${ }^{24}$ [traduction libre] (2010, p. 19).

Comment sortir de ces dichotomies qui ne nous permettent pas de saisir la complexité de la situation des femmes?

Les travaux de Crenshaw (1991) sur l'intersectionnalité et ceux de Collins (2000) sur les matrices de domination sont éclairants alors qu'ils insistent sur l'importance de replacer le sujet (structuré par le genre, la race, la classe sociale, etc.) dans son environnement pour ainsi dégager les différents lieux où le sujet expérimente et résiste aux oppressions. Dans le cas qui m'intéresse ici, cela implique de poser les réalités des femmes dans une matrice qui tient compte de divers contextes. D'abord le contexte international, le macrosystème (conflit israélo-palestinien, politiques internationales de migration et droit international), puis le contexte national libanais (facteurs sociopolitiques et conditions économiques du pays) et enfin le contexte 
personnel (valeurs personnelles, familiales et communautaires des femmes, de même que les facteurs socioéconomiques qui influencent leurs décisions). L'analyse intersectionnelle rééquilibre donc une grille de lecture axée sur les résistances des femmes en inscrivant leurs réalités dans une matrice qui montre les effets interactifs des divers systèmes d'oppression. C'est là où réside le défi : articuler la matrice d'oppressions et de privilèges et démontrer son applicabilité. Ceci dit, même si des critiques ont été formulées sur cette méthode, je crois qu'un tel travail est important tant qu'il est utilisé « tel un catalyseur chimique afin de mettre en lumière les relations de pouvoir, identifier leur position, connaître leurs applications et leurs méthodes $»^{25}$ [traduction libre] (Abu-Lughod, 1990, p. 42). Ici, une telle analyse permet d'identifier les conditions, les structures et les forces qui contribuent à maintenir ces femmes dans une situation de pauvreté et ainsi de révéler des pistes d'action pour y remédier.

Le concept de «matrice de domination» de Collins (2000) est utile pour articuler les rapports de pouvoir: dégager différentes structures qui, par leur croisement, contribuent tantôt à créer, tantôt à maintenir des situations d'oppression, de domination ou encore de privilège et de résistance. Selon cette auteure, nous aurions tendance à nous identifier à une oppression - celle dont nous avons fait l'expérience et à considérer toutes les autres formes d'oppression comme moins importantes; dans notre esprit, cette oppression prend une place maîtresse. En ce qui me concerne, le genre occupe une place importante dans la, ou plutôt ma matrice d'oppressions, mais pour plusieurs Palestiniennes, ce n'est pas le cas. J'ai pu observer que l'oppression liée à leur statut de réfugiées, de réfugiées palestiniennes, joue un rôle primordial pour elles. Pour lutter contre les inégalités liées à ce statut, elles sont plusieurs à avoir priorisé la lutte nationale (une lutte au «nous »). Comme le précise à nouveau Collins, on doit rester vigilant - particulièrement en tant qu'outsider - au fait que la notion de vécu expérientiel peut privilégier l'expérience et la connaissance individuelles au détriment du point de vue collectif. Ceci me renvoie à une question soulevée précédemment: que me révèle mon intérêt constant à rechercher le «je» dans le discours des femmes? Il y avait un objectif théorique à cet exercice : examiner les rapports de pouvoir pour vérifier s'il y a, ou non, domination du « nous » sur le «je ». Mon idée n'était pas de faire la promotion $\mathrm{du}$ «je » au détriment du «nous », malgré qu'il soit difficile - je l'avoue - de ne pas tomber dans un tel piège... Ce constat a suscité chez moi une série d'interrogations : les subalternes peuvent-elles parler? Estce que j'écoute « vraiment » les femmes et la façon dont elles se racontent? Ma grille de lecture féministe postcoloniale - avec son analyse, ses concepts, ses schémas, etc. me permet-elle de comprendre les femmes? Ai-je bien compris et appliqué cette grille ou bien suis-je confrontée à ses limites? 


\section{Réflexions finales}

En m'appuyant sur la démarche réflexive que j'ai réalisée dans le cadre de ma thèse, j'ai tenté dans le présent article de montrer comment peut se négocier dans le milieu de recherche - et même au-delà - une position de femme, étrangère, non palestinienne, non musulmane et déterminée à « porter les voix » d'une communauté « Autre ». Tout au long de mon processus, j'ai toujours cherché à comprendre l'expérience des réfugiées palestiniennes, et cela, grâce à un travail continu mais combien déstabilisant, voire douloureux, de réflexivité. L'accès aux voix de ces femmes n'est possible qu'au prix d'une vigilance vis-à-vis à la fois du milieu de recherche et du produit textuel. En effet, représenter l'Autre, "Autre pour lequel on parle et dont on revendique de représenter ce qu'il ressent et ce qu'il représente » (Ghasarian, 2004, p. 223), doit se faire à travers un processus négocié de collaboration et de dialogue avec cet Autre et par une compréhension de son positionnement en tant que chercheure. Ce n'est qu'à ce prix qu'on pourra échapper à la violence de la représentation (Spivak, 1988)...

\section{Notes}

${ }^{1}$ Mot arabe signifiant « étrangère ».

2 «If I failed to publicize their message, I would be breaking the implicit condition upon which they offered their stories... » (Sayigh, 1996, p. 161).

${ }^{3}$ Afin de s'immerger dans la réflexivité et les questionnements de la chercheure principale, il a été convenu d'écrire cet article sous la forme d'un récit au «je », narré par Roxane Caron. Dominique Damant, coauteure, a longuement participé aux discussions et réflexions qui sont au cœur même de cet article. Quant à Catherine Flynn, elle s'est ajoutée à l'équipe pour la révision de l'article.

4 «Quel est ton nom? D'où viens-tu?»

${ }^{5}$ Salutations d'usage : «Bonjour, bienvenue, que la paix soit avec vous. »

${ }^{6}$ Four à pain traditionnel.

${ }^{7}$ L'appel à la prière.

${ }^{8}$ Le camp palestinien de Bourj el Barajneh

9 Je suis retournée dans le même logement que j'avais habité, donc avec le même voisinage qui n'avait que très peu changé...

10 Je dis «désiraient» parce que plusieurs femmes étaient enthousiastes à l'idée de me « raconter leur histoire » et non de répondre à un questionnaire.

${ }^{11}$ Pour la collecte de données, j'ai habité le camp palestinien de Bourj el Barajneh pour une période d'un peu plus d'un an et demi (2009-2011).

12 Office de secours et de travaux des Nations Unies pour les réfugiés de Palestine dans le Proche-Orient. 
${ }^{13}$ Ce terme réfère à la catastrophe ou au désastre lié à la perte de la terre de Palestine. On l'attribue à Constantin Zureik (1919-2000), une des figures intellectuelles du nationalisme arabe.

${ }^{14}$ Les travaux de Rosemary Sayigh (1994, 1996, 1998, 2007a, 2007b) ont été précurseurs en ce qui a trait aux écrits sur l'expérience des femmes réfugiées palestiniennes : leurs pratiques, leurs mémoires, leur engagement et leur rôle dans la lutte nationale palestinienne. Voir aussi Julie Peteet (1991), Nahla Abdo et Ronit Lentin (2002) et Stéphanie Latte Abdallah (2004) (liste non exhaustive).

15 «Can the subaltern speak? » (Spivak, 1988).

${ }^{16}$ Ici, je fais le choix de garder les termes outsider/insider, termes anglais largement utilisés dans la littérature francophone pour désigner le statut (ou perspective) qu'a le chercheur avec le terrain de recherche.

${ }^{17}$ Il est à noter que les verbatims originaux sont en anglais. Pour les biens du présent article, ils ont été traduits en français.

18 Je fais référence ici aux deuxièmes entretiens, soit les entretiens rétroactifs, que j'ai réalisés avec 12 participantes de mon échantillon initial, après avoir fait une analyse préliminaire de mon corpus.

19 «self-definition is the first step to empowerment : if a group is not defining itself, then it is being defined by and for the use of other » (Collins, 2000, p. 101).

20 "I feared that the national crisis and nationalistic discourse would so dominate what respondents would [...] recount that their experiences of gender and sexuality would be eliminated ( Sayigh, 1996, p. 159).

${ }^{21}$ «life-story may contribute to a sense of the person as its center as an isolated individual » (Abu-Lughod, 1993, p. 31).

${ }^{22}$ Sayigh (2007b) s'est intéressée à la structure des récits de vie de réfugiées palestiniennes au Liban.

${ }^{23}$ «Can the subaltern speak?» (Spivak, 1988).

24 "With their eagerness to "give voice" and inspire activism by finding or reinventing traditions of resistance, resistance-oriented approaches, biased by a certain romanticism, significantly impoverish the analysis of power - researchers being too concerned to find resisters and not attentive enough to explaining the workings of power. What is therefore needed is a way to keep the intersectional analysis embedded in postcolonial readings while at the same time dissociating the interpretation of veiled women's agency from the doxa of resistance » (Bilge, 2010, p. 19).

${ }^{25}$ " as a chemical catalyst so as to bring to light power relations, locate their position, find out their points of application and the methods used» (Abu-Lughod, 1990, p. 42). 


\section{Références}

Abdo, N., \& Lentin, R. (2002). Women and the politics of military confrontation. Palestinian and Israeli gendered narratives of dislocation. New York, NY: Berghahn.

Abu-Lughod, L. (1990). The romance of resistance : Tracing transformations of power through Bedouin women. American Ethnologist, 17(1), 41-55.

Abu-Lughod, L. (1993). Writing women's worlds : Bedouin stories. Berkeley, CA : University of California Press.

Abu-Lughod, L., \& Sa'di, A. H. (2007). Nakba. Palestine, 1948, and the claims of memory. New York, NY : Columbia University Press.

Agamben, G. (1999). Ce qui reste d'Auschwitz. Paris : Rivages.

Anthias, F. (2005). Social stratification and social inequality: Models of intersectionality and identity. Dans F. Devine, R. Crompton, M. Savage, \& J. F. Scott (Éds), Rethinking class : Culture, identities and lifestyles (pp. 24-45). New York, NY : Palgrave Macmillan.

Bilge, S. (2010). Beyond subordination and resistance : An intersectional approach to the agency of veiled muslim women. Journal of Intercultural Studies, 31(1), 9-28.

Caron, R. (2007). Les stratégies de survie de femmes réfugiées palestiniennes du camp de Bourj el Barajneh au Liban (Mémoire de maîtrise inédit). Université Laval, Québec, QC.

Caron, R. (2012). Entre refuge et exil. L'expérience de femmes palestiniennes du camp de Bourj el Barajneh (Thèse de doctorat inédite). Université de Montréal, Montréal, QC.

Collins, P. H. (2000). Black feminist thought: Knowledge, consciousness, and the politics of empowerment. New York, NY : Routledge.

Crenshaw, K. (1991). Mapping the margins: Intersectionality, identity, politics and violence against women of color. Standford Law Review, 43(6), 1241-1299.

De Gaulejac, V. (1987). La névrose de classe. Paris : Éditions Hommes et Groupes.

Fainzang, S. (2004). De l'autre côté du miroir. Réflexions sur l'ethnologie des anciens alcooliques. Dans C. Ghasarian (Éd.), De l'ethnographie à l'anthropologie réflexive. Nouveaux terrains, nouvelles pratiques, nouveaux enjeux (pp.63-71). Paris : Armand Colin.

Fisk, R. (1990). Pity the nation. Lebanon at war. Londres : Deutsch. 
Ghasarian, C. (2004). Sur les chemins de l'ethnographie réflexive. Dans C. Ghasarian (Éd.), De l'ethnographie à l'anthropologie réflexive. Nouveaux terrains, nouvelles pratiques, nouveaux enjeux (pp. 5-34). Paris : Armand Colin.

Godelier, M. (2004). Briser le miroir du soi. Dans C. Ghasarian (Éd.), De l'ethnographie à l'anthropologie réflexive. Nouveaux terrains, nouvelles pratiques, nouveaux enjeux (pp. 193-212). Paris : Armand Colin.

Guillaumin, C. (1992). Sexe, race, et pratique de pouvoir : L'idée de nature. Paris : Côté femmes.

Harper, E. (2012). Regards sur l'intersectionnalité. Centre de recherche interdisciplinaire sur la violence familiale et la violence faite aux femmes. Collection études et réflexion, $44 . \quad$ Repéré à https://www.criviff.qc.ca/sites/criviff.qc.ca/files/publications/pub_06112012_8335 2.pdf

Hasso, F. S. (2000). Modernity and gender in Arab accounts of the 1948 and 1967 defeats. International Journal of Middle East Studies, 32(4), 491-510.

Holland, J., \& Ramazanoglu, C. (2002). Feminist methodology: Challenges and choices. Londres : Sage.

Holt, M. (2011). A world of movement. Memory and reality for Palestinian women in the camps of Lebanon. Dans S. Hanafi, \& A. Knudsen (Éds), Palestinian refugees. Identity, space and place in the levant (pp. 180-192). Londres: Routledge.

Humm, M. (1995). The dictionary of feminist theory. Columbus, OH : Ohio State University Press.

Kandiyoti, D. (1996). Gendering in the middle east : Emerging perspectives. Syracuse, NY : Syracuse University Press.

Latif, N. (2008). Space, power and identity in a refugee camp. Asylon, 5. Repéré à : http://www.reseau-terra.eu/article800.html

Latte Abdallah, S. (2004). Femmes réfugiées palestiniennes. Paris: Presses universitaires de France.

Maynard, M. (1994). Methods, practice and epistemology : The debate about feminism and research. Dans M. Maynard, \& J. Purvis (Éds), Researching women's lives from a feminist perspective (pp. 10-26). Londres : Taylor and Francis.

Mohanty, C. T. (1988). Under western eyes: Feminist scholarship and colonial discourses. Feminist Review, 30, 65-88.

Orofiamma, R. (2008). Les figures du sujet dans le récit de vie. En sociologie et en formation. Informations sociales, 1(145), 68-81. 
Peteet, J. (1991). Gender in crisis : Women and the palestinian resistance movement. New York, NY : Columbia University Press.

Rabinow, P. (1988). Un ethnologue au Maroc. Réflexions sur une enquête de terrain. Paris : Hachette.

Rose, D. (2002). Retour sur les méthodologies de recherche féministe [Document de travail]. Ottawa : Condition Féminine Canada.

Sayigh, R. (1994). Too many enemies: The Palestinian experience in Lebanon. Londres : Zed Books

Sayigh, R. (1996). Researching gender in a Palestinian camp : Political, theoretical and methodological issues. Dans D. Kandiyoti (Éd.), Gendering in the middle east: Emerging perspectives (pp. 145-168). Syracuse, NY : Syracuse University Press.

Sayigh, R. (1998). Palestinian camp women as tellers of history. Journal of Palestine Studies, 27(2), 42-58.

Sayigh, R. (2007a). The Palestinians: From peasants to revolutionaries ( $2^{\mathrm{e}}$ éd.). New York, NY : Zed Books.

Sayigh, R. (2007b). Women's nakba stories. Between being and knowing. Dans L. Abu-Lughod, \& A. H. Sa'di (Éds), Nakba. Palestine, 1948, and the claims of memory (pp. 135-160). New York, NY : Columbia University Press.

Siran, J. L. (1994). Les énoncés ne sont pas des choses mais des événements. Journal des anthropologues, 57-58, 107-117.

Spivak, G. C. (1988). Can the subaltern speak? Dans L. Grossberg, \& C. Nelson (Éds), Marxism and the interpretation of culture (pp. 271-313). Champaign, IL: University of Illinois Press.

Roxane Caron, Ph. D., est professeure adjointe à l'École de travail social de l'Université de Montréal. Sa recherche doctorale portait sur l'expérience d'exil de femmes réfugiées palestiniennes au Liban. Ses travaux s'ancrent dans une approche qualitative, tout particulièrement l'ethnographie et le récit de vie. Elle s'intéresse aux expériences des femmes réfugiées.

Dominique Damant, Ph. D., est professeure honoraire à l'École de travail social de l'Université de Montréal. Elle a dirigé de nombreux projets de recherche subventionnés autour de la question de la violence faite aux femmes, de la violence perpétrée par les femmes et de la maternité. Plus récemment, elle s'est intéressée à la maternité en contexte de violence chez les femmes racisées et autochtones et à la violence structurelle. 
Catherine Flynn, Ph. D., est professeure substitut au Département de psychosociologie et de travail social de l'Université du Québec à Rimouski. Elle s'intéresse aux violences conjugales et aux violences faites aux femmes dans une perspective intersectionnelle. Ces travaux portent principalement sur l'expérience des jeunes femmes en situation d'itinérance et sur le développement d'interventions féministes intersectionnelles. 